\title{
Time-course of systems consolidation and fear memory generalization in adolescent rats of different sex
}

Ana P. Crestani ${ }^{1,4^{*}}$, Fernanda N. Lotz ${ }^{1,3^{*}}$, Mirelle A. Casagrande ${ }^{2,3}$, Bruno Popik ${ }^{2,3}$, Kétlyn T. K. Guerra $^{1,3}$, Lucas de Oliveira Alvares ${ }^{2,3}$, Jorge A. Quillfeldt ${ }^{1,3, \#}$

1 Psychobiology and Neurocomputation Laboratory, Biophysics Department, Biosciences Institute, Federal University of Rio Grande do Sul, Porto Alegre - RS, Brazil.

${ }^{2}$ Neurobiology of Memory Laboratory, Biophysics Department, Biosciences Institute, Federal University of Rio Grande do Sul, Porto Alegre - RS, Brazil.

${ }^{3}$ Graduate Program in Neuroscience, Institute of Health Sciences, Federal University of Rio Grande do Sul, Porto Alegre - RS, Brazil.

${ }^{4}$ Ribeirão Preto Medical School, Neuroscience and Behavioral Sciences Department, University of São Paulo, Ribeirão Preto - SP, Brazil.

* These authors contributed equally to this work.

\# Corresponding authors.

\section{Corresponding authors:}

Jorge Alberto Quillfeldt, Laboratório de Psicobiologia e Neurocomputação, Departamento de Biofísica, Instituto de Biociências, Universidade Federal do Rio Grande do Sul (UFRGS), Av. Bento Gonçalves 9500, Prédio 43422, Sala 208, CEP 91.501-970, Porto Alegre, Rio Grande do Sul, Brasil. e-mail: quillfe@ufrgs.br

Ana Paula Crestani, Ribeirão Preto Medical School, Neuroscience and Behavioral Sciences Department, University of São Paulo, Av. Bandeirantes 3900, CEP: 14049-900, Ribeirão Preto - SP, Brazil. e-mail: crestaniap@usp.br 


\begin{abstract}
Systems consolidation is a time-dependent process in which the retrieval of contextual memories becomes progressively independent from the hippocampus and more reliant on cortical structures. One qualitative consequence, supposed to be causally related, is the progressive loss of memory precision. Memory generalization, however, is a much more complex phenomena, and may take place much faster in adult animals, for instance, in response to changes in the available levels of sex hormones. In this sense, females are known to display a generalized memory earlier than males. Sex differences might have an important role in the modulation of mnemonic processes, and despite being of paramount importance for the understanding of the cognitive dynamics of juveniles, it remains poorly investigated. Here, we studied adolescent rats (P42-49) of different sex comparing the natural time-course of systems consolidation as verified both by the hippocampus-dependency and the onset of memory generalization. Contextual fear discrimination was quantified at different time-points after learning (2, 7, 14, 21 and 28 days). Our results demonstrated that, contrary to what is observed in adults, memory generalization occurs earlier in adolescent males (14 days) than in females (28 days). During adolescence, females display a higher mean discrimination index at all time-points, suggesting that they retain a more detailed memory. Likewise, pre-test pharmacological inactivation of the hippocampus (with $\mathrm{GABA}_{\mathrm{A}}$ agonist muscimol) was able to impair memory retrieval in females, but not in males, 14 days after training. These results support a causal relationship between memory generalization and retrieval independence from the hippocampus in male adolescent rats. However, both aspects of the systems consolidation process appear to be distinctly modulated in animals of different sex, with males (and ovariectomized females) enduring an accelerated onset of both memory precision loss and the hippocampus independence compared to females, suggesting a clear role for gonadal hormones in this cognitive corticalization process.
\end{abstract}

Keywords: memory, generalization, systems consolidation, hippocampus, sex 


\section{INTRODUCTION}

The ability to make associations between places and events emerges already in infancy [1] and predicting the safety of future experiences provides adaptive advantages - a capacity that might also have had evolutionary consequences [2]. Several important modifications occurring in the nervous and endocrine systems during ontogeny, may have had considerable influence on how much detailed memories can be produced, and to what extent animals can discriminate between similar, yet different environments [3].

Previous studies have demonstrated that memory loses precision in a contextual discrimination task as the interval between training and test increases: as time after training passes, it becomes more generalized [4-6]. Modifications in brain regions that support memory retrieval also occur over time, with the memory trace retrieval becoming progressively independent from the hippocampus and reliant on cortical areas [7-13]. Despite not fully understood, systems consolidation appear to express itself in two dimensions, one anatomical - the gradual independence from the hippocampus - and the other, qualitative - the progressive inability to discriminate between similar contexts, i.e., memory generalization $[4,6,14]$.

Most experiments evaluating memory generalization and systems consolidation are performed in adult male rodents, obtaining a time-course of generalization of around one month. Adult female rats, however, appear to generalize memory faster and in an estradiol-dependent manner $[17,18]$. Gonadal hormones are produced in a sex-specific manner throughout adolescence [19-21], influencing behavioral performance [3,22-25]. Accordingly, brain structures critically involved in learning and memory processes such as the hippocampus, amygdala, and medial prefrontal cortex, undergo extensive morphological and functional remodeling during this period $[22,26]$.

Considering the importance of generalization in the face of danger-related situations and the well-known behavioral differences between adolescent males and females in relation to threat exposure or risk-taking [25,26], it is reasonable to suppose differences could also be observed in the time-course of memory generalization observed along the systems consolidation process. Thus, we investigated contextual fear discrimination in adolescent rats of different sex at different training-test intervals $(2 \mathrm{~d}, 7 \mathrm{~d}, 14 \mathrm{~d}, 21 \mathrm{~d}$ and 28d). Additionally, hippocampal pharmacological inactivation during retrieval was used to access hippocampus dependency. To verify for possible 
female-related hormonal changes on the observed effects, ovariectomized females were also investigated.

\section{MATERIAL AND METHODS}

\section{Animals}

Subjects were the offspring of Wistar rats obtained from the University's breeding colony. Male and female pups were bred in our housing facility, weaned on postnatal day 21 (P21) and housed with same-sex littermates, up to 5 per cage, until contextual fear conditioning training was performed during their late adolescence (P42-P49). Animals were kept on a 12/12h dark/light cycle and had food and water available ad libitum. Experiments were performed in accordance with National Animal Care Legislation and Guidelines (Brazilian Law 11794/2008) and approved by the University's Ethics Committee (project \# 23957 and 27143).

\section{Behavioral Procedure}

Contextual Fear Conditioning: All subjects underwent training in the contextual fear conditioning (CFC). The CFC chamber consisted of an illuminated Plexiglas box $(25.0 \times 25.0 \mathrm{~cm}$ with a grid floor of parallel $0.1 \mathrm{~cm}$ caliber stainless steel bars spaced $1.0 \mathrm{~cm}$ apart) with fan background noise. In the conditioning session, rats were placed in the chamber for $3 \mathrm{~min}$, after which they received two 2 -sec $0.7 \mathrm{~mA}$ footshocks separated by a 30-sec interval. Animals were kept in the conditioning environment for an additional $30 \mathrm{sec}$ before they were returned to their homecages. Experiments were divided into (i) time-course of the onset of memory generalization in adolescent males and females, (ii) hippocampal dependency for memory retrieval in adolescent animals of both sex and (iii) generalization and hippocampal dependency for retrieval in ovariectomized females, as better detailed below.

\section{First set of experiments: Contextual fear memory generalization time-course}

In order to investigate fear memory expression in different, yet similar contexts, adolescent male and intact, naturally cycling female rats were tested in the conditioned or in a novel context on days $2,7,14,21$ or 28 after training. The novel context was a rectangular box $2 / 3$ the size of the 
conditioning context with a smooth floor, without background fan noise and dimmer overhead lighting; the chamber was located in an adjacent area with different spatial cues. Freezing behavior was registered in real-time during a 4-min test by an experienced and blinded observer, and was defined as the absence of all movements except those related to breathing.

\section{Second set of experiments: Hippocampal dependency for memory retrieval}

Male and female animals were submitted to stereotaxic surgery to allow for subsequent pharmacological inactivation of the hippocampus during memory retrieval. The test session (with hippocampal inactivation) was performed at the time-point when memory generalization in males had been observed (14 days after training) to evaluate whether the loss of precision was correlated with hippocampus-independence for memory retrieval. Female subjects were also tested at the same time-point without monitoring estrous cycle stage.

Stereotaxic surgery and cannulae placement: Rats were anesthetized with an intraperitoneal injection of ketamine/xylazine (75 and $10 \mathrm{mg} / \mathrm{Kg}$, respectively) and bilaterally implanted with guide cannulae (22-gauge) aimed at the dorsal hippocampus at the following coordinates: AP -3.0 $\mathrm{mm}$ (from Bregma), LL $\pm 2.0 \mathrm{~mm}, \mathrm{DV}-1.5 \mathrm{~mm}$ (males) and DV -1.4 mm (females) - DV positioned $1.0 \mathrm{~mm}$ above each structure [27]. Surgery was performed 7-8 days after training. After surgery, animals were given 6-7 days to recover before being submitted to the behavioral procedure (14 days after training).

Drug: Muscimol ( $1 \mu \mathrm{g} / \mu 1$; Research Biochemicals International), a GABA $\mathrm{A}_{\mathrm{A}}$ agonist, was dissolved in phosphate-buffered saline (PBS) and bilaterally infused into dorsal hippocampus 15min before test. Vehicle groups underwent the same manipulation but received PBS.

Intracerebral infusion: At the time of infusion, a 27-gauge infusion needle was inserted into each guide cannula, with its tip protruding $1.0 \mathrm{~mm}$ beyond the tip of the cannula and aimed at the dorsal hippocampus. A volume of $0.5 \mu 1$ was bilaterally infused at a slow rate $(20 \mu 1 / h)$, and the needle removed only after waiting an additional $30 \mathrm{sec}$.

Histology: Cannula position was verified at the end of each experiment. Brains were removed and immersed in a fixation solution of $20 \%$ sucrose and $4 \%$ paraformaldehyde. Brains were then frozen 
and sliced (50 $\mu \mathrm{m}$ coronal sections) using a cryostat. Sections were stained with cresyl violet and subsequently examined to verify the location of the cannulae. Only animals with correct cannulae placements were considered in the statistical analysis.

Third set of experiments: Memory generalization and hippocampal dependency in ovariectomized females

A group of ovariectomized (OVX) females were tested 14 days after training in the conditioned or in the novel context to evaluate memory expression compared to the normal, intact females above.

Ovariectomy procedure: prepubescent rats (P30-33) were anesthetized with an IP combination of ketamine/xylazine (75 and $10 \mathrm{mg} / \mathrm{Kg}$, respectively). A ventral midline skin incision approximately $1.5 \mathrm{~cm}$ long was made with subsequent removal of the ovaries, after which the uterine tubes were ligated. Afterwards, ventral muscles and skin were sutured and animals received post-surgery treatment with an analgesic, namely Acetaminophen $200 \mathrm{mg} / \mathrm{ml}$ (brand name Tylenol®) diluted in water.

Finally, another group of OVX females was used to evaluate whether the observed memory performance (expected to be generalized) was dependent on the hippocampus. In this case, this group was implanted with cannulae in the dorsal hippocampus 7-8 days after conditioning and tested on day 14. Cannulae placements were checked as described above.

\section{Statistical analysis}

In experiment 1 , three variables (sex, time and context) were studied, however, considering that males of the same age of females naturally differ in their behavioral performance, and forgetting might be considered negligible along this 28 days period, we did not expect to obtain the exact same mean in all 20 experimental groups (i.e., we could not suppose a null hypothesis in which all means are equal), thus, instead of using ANOVA, data was analyzed by Student's t-test for independent samples only, the same parametric test used in experiments 2 and 3. After checking for normality (Kolmogorov-Smirnov Test) and homoscedasticity (Levene test), means from each pair of training vs. novel context were separately compared in experiments 1 and 3 (Figure 1.A-J). In experiment 2, the same analysis was applied to compare vehicle vs. drug groups. Significance level was set at $\mathrm{P}<0.05$. 


\section{RESULTS}

\subsection{Time-course of fear memory generalization in adolescent males and females}

To test whether sex differences during adolescence influence contextual discrimination, separate groups of adolescent male or female rats were trained in the contextual fear conditioning and tested in either the conditioned, or the novel environment 2, 7, 14, 21, or 28 days later.

Adolescent male rats discriminated between contexts when tested 2 (Fig. 1A; $t_{(15)}=$ 2.275; $\mathrm{P}=0.038 ; \mathrm{N}=8,9)$ or 7 days $\left(\right.$ Fig. $\left.1 \mathrm{~B} ; \mathrm{t}_{(25)}=3.42 ; \mathrm{P}=0.0022 ; \mathrm{N}=13,14\right)$ after training, but not at $14\left(\right.$ Fig. 1C; $\left.\mathrm{t}_{(24)}=0.9099 ; \mathrm{P}=0.3719 ; \mathrm{N}=13,13\right), 21\left(\right.$ Fig. 1D; $\mathrm{t}_{(10)}=1.422 ; \mathrm{P}=0.1854 ; \mathrm{N}=$ $6,6)$ or $28\left(\right.$ Fig. 1E; $\left.\mathrm{t}_{(14)}=1.643 ; \mathrm{P}=0.1227 ; \mathrm{N}=8,8\right)$ days. On the other hand, adolescent females discriminated more precisely between contexts 2 (Fig. 1F; $\mathrm{t}_{(11)}=3.893 ; \mathrm{P}=0.0025 ; \mathrm{N}=6,7$ ), 7 (Fig. 1G; $\left.\mathrm{t}_{(12)}=6.502 ; \mathrm{P}=0.0001 ; \mathrm{N}=7,7\right), 14$ (Fig. 1H; $\mathrm{t}_{(14)}=5.073 ; \mathrm{P}=0.002 ; \mathrm{N}=8,8$ ) and 21 (Fig. 1I; $\left.\mathrm{t}_{(16)}=2.556 ; \mathrm{P}=0.0212 ; \mathrm{N}=8,10\right)$ days posttraining, and showed generalized memory on day 28 (Fig. 1J; $\left.\mathrm{t}_{(17)}=1.812 ; \mathrm{P}=0.0878 ; \mathrm{N}=9,10\right)$. Also, females had a higher discrimination index when compared to males at all the time-points tested, as can be noted in Fig. 1M.

Therefore, we found a systematic increase in generalization over time with fear generalization occurring earlier in adolescent males than in females. Males lose memory precision 14 days after training (Fig. 1K) whereas females maintain memory precision up to 28 days (Fig. 1L). The discrimination index suggests that adolescent females distinguish better in all the assessed time-points (Fig. 1M). 

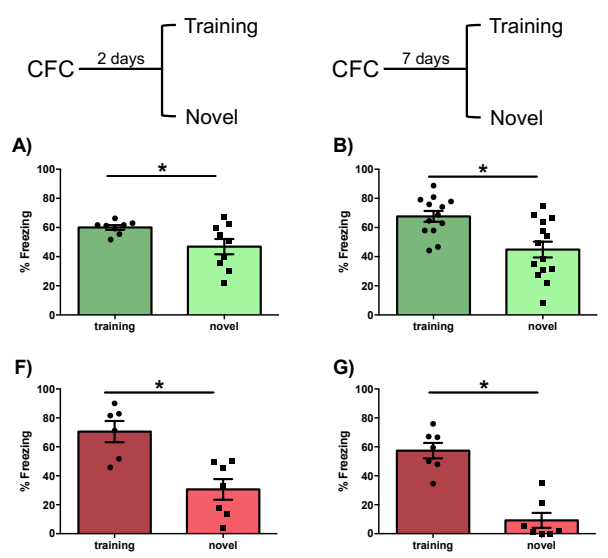

K)
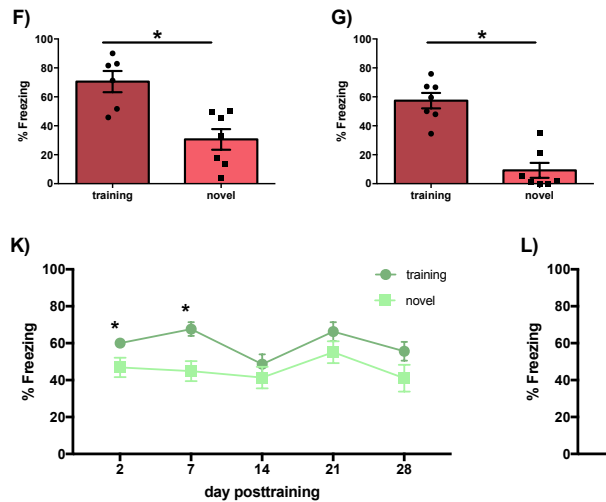

L)

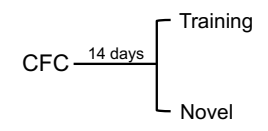

C)
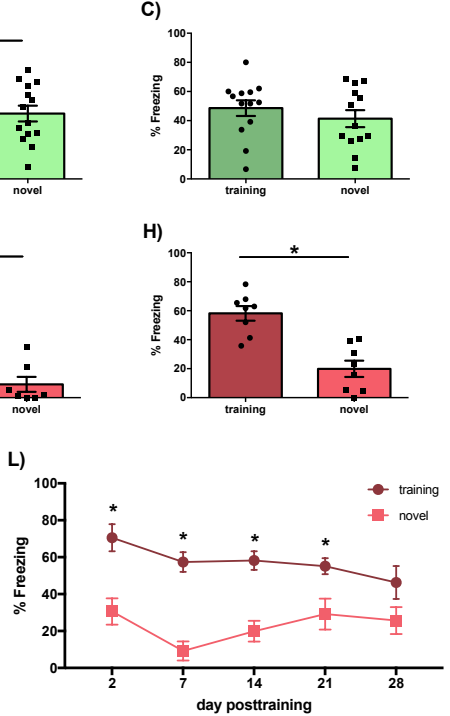
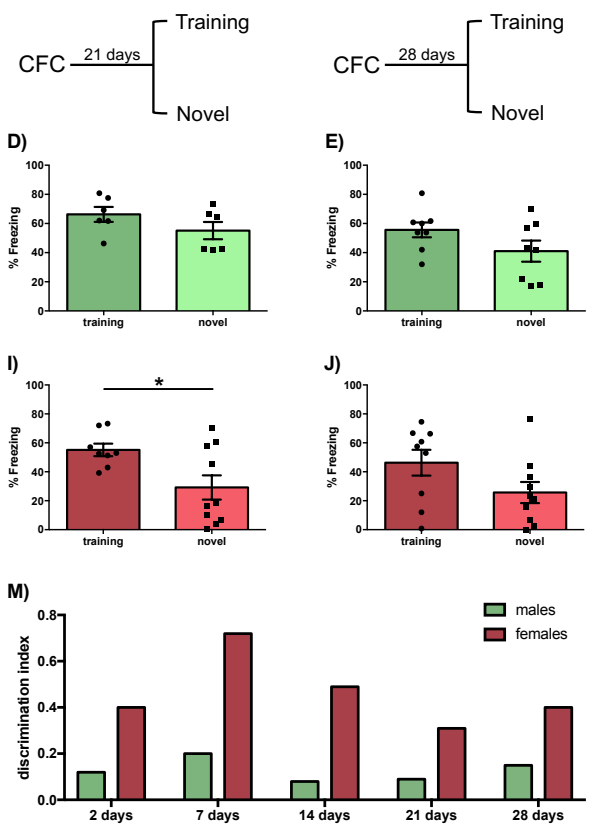

Figure 1. Time-course of fear memory generalization in adolescent male and female rats. Different groups of adolescent male rats were tested either in the training, or the novel context at 2 days (A), 7 days (B), 14 days (C), 21 days (D) or 28 days (E) after training: male rats discriminated between contexts when tested 2 and 7 days after training, but not at 14, 21 and 28 days. Females were tested at the same time-points and presented precise discrimination at 2 days $(\mathbf{F}), 7$ days $(\mathbf{G}), 14$ days $(\mathbf{H})$ and 21 days $(\mathbf{I})$, showing memory generalization only around day $28^{\text {th }}(\mathbf{J})$. Groups tested at the same time-point after training, for each sex, were compared using Student's t-test. Color code: training context is represented by dark green (males) and dark red (females) while novel context is represented by light green (males) and light red (females). (K and L) For a better visualization, the same results of previous bar graphs (A-J) are now presented as a line graph over time, respectively, (K) for males, and (L) for females. (M) Histogram showing a mean discrimination index defined as (\% freezing in the training context minus the $\%$ freezing in the novel one) / (\% freezing in the training context $(\mathrm{T})+\%$ freezing in the novel context) for males and females in all the investigated time-points. Percentage of freezing was expressed as Mean \pm SEM. $\left(^{*}\right) \mathrm{P}<0.05$ (see text for more details).

\subsection{Memory retrieval 14 days after training is dependent on hippocampus in females, but not in males}

Transformation theory predicts that contextual memories are specific soon after training and require the hippocampus for retrieval while remote memories become more generic as they are permanently stored in the cortex $[28,29]$.

To verify for memory retrieval hippocampus-dependence, dorsal hippocampus was inhibited with the infusion of GABAa agonist muscimol prior to testing (in the training context). The specific time-point chosen to investigate memory retrieval was 14 days after training. Our aforementioned result demonstrated that at this time-point, memory becomes generalized in males. but still retains some precision in females (Fig. 1). Our prediction was that memory retrieval would not be affected by hippocampus inactivation in males, whereas memory would be impaired in 
females: and that was confirmed, muscimol was ineffective upon memory retrieval in males (Fig. 2A; $\left.\mathrm{t}_{(12)}=0.3309 ; \mathrm{P}=0.7464 ; \mathrm{N}=7,7\right)$, showing that memory generalization is concomitant with neuroanatomical systems consolidation. On the other hand, muscimol blocked memory retrieval in females (Fig. 2B; $\left.\mathrm{t}_{(18)}=4.008 ; \mathrm{P}=0.0008 ; \mathrm{N}=10,10\right)$ proving that memory, when precise, is still dependent on the hippocampus.

A)
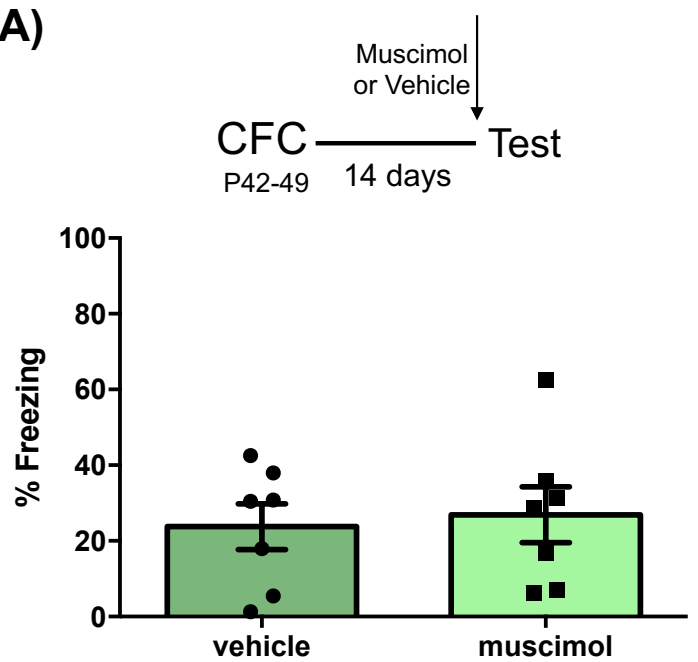

B)
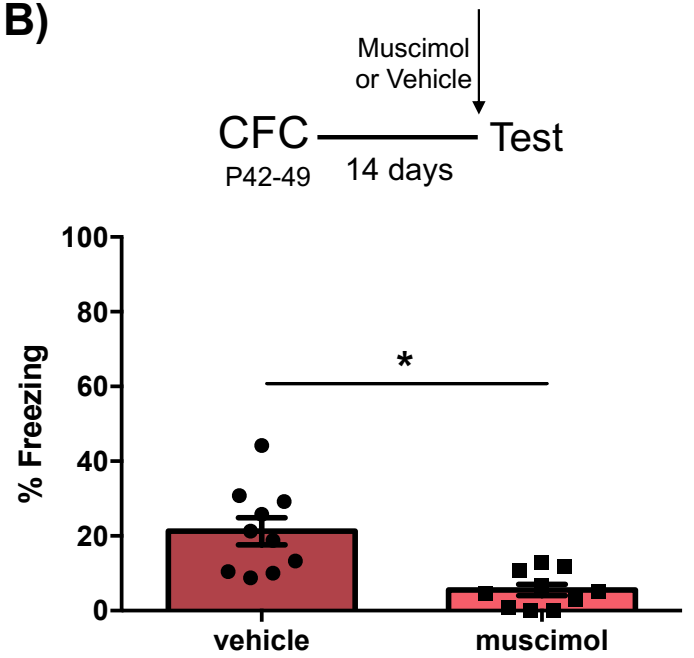

Figure 2. Memory retrieval 14 days after training is dependent on hippocampus in females, but not in males. GABAa agonist muscimol was infused in the dorsal hippocampus prior to the retrieval test in the training context. Muscimol did not inhibit memory retrieval in males (A), but impaired memory retrieval in females (B). Groups were compared using Student's $t$ test for independent samples. Percentage of freezing was expressed as Mean \pm SEM. $\left({ }^{*}\right)$ $\mathrm{P}<0.05$ (see text for more details).

\subsection{Generalization and hippocampus dependency in ovariectomized females}

Our last set of experiments tested whether female hormones could be involved in the maintenance of memory precision found in females.

Indeed, ovariectomized females generalize memory earlier, showing a dynamic of generalization similar to males (Fig. 3A; $\left.\mathrm{t}_{(18)}=1.607 ; \mathrm{P}=0.1255 ; \mathrm{N}=10,10\right)$. In this case, hippocampal inactivation also did not impair memory retrieval (Fig. 3B; $\mathrm{t}_{(12)}=1.92 ; \mathrm{P}=0.0789$; $\mathrm{N}=6,8)$.

These results demonstrate that ovariectomy accelerates memory generalization and hippocampus independence, and point to a possible role of female hormones in the maintenance of memory quality and in delaying systems consolidation, as proposed by others. 

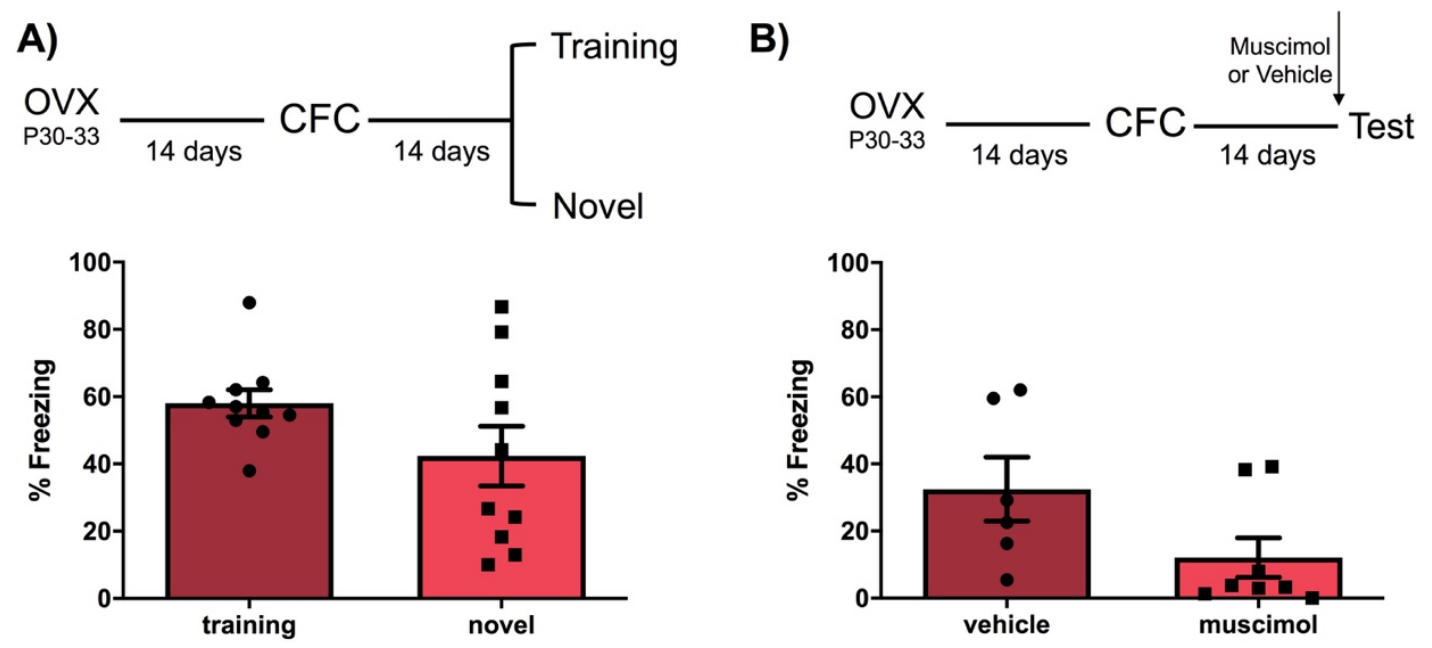

Figure 3. Ovariectomy anticipates memory generalization and hippocampus-independence in female rats. (A) Ovariectomized females generalize memory earlier, showing a dynamic of generalization similar to males. (B) Moreover, hippocampal inactivation did not impair memory retrieval. Groups were compared using Student's t test for independent samples. Percentage of freezing was expressed as Mean \pm SEM. Differences were not statistically significant (see text for more details).

\section{$\underline{\text { Discussion }}$}

Our results indicate that fear memory generalization due to systems consolidation display sex dimorphism, taking place earlier in adolescent males (Fig. 1A-E;K) than in females (Fig. 1F-J;L). Adolescent males demonstrated memory generalization from 14 days after training (Fig. 1C) - which is earlier than adults use to show in the experience of our and other labs (see below) - whereas adolescent females kept memory precision up to 28 days (Fig. 1J). The memory generalization displayed by males 14 days after conditioning was shown not to depend on the hippocampus, since concomitant infusion of muscimol was ineffective (Fig. 2A), which is also earlier than what is observed in adult rats. At this time-point, while control females memory still display a precise context discrimination as well as hippocampus-dependency (Fig. 2B), ovariectomized subjects appear to have accelerated the expression both of context generalization (Fig. 3A) and hippocampus independence (Fig. 3B).

Detailed memories are known to depend upon the hippocampus [4[4,6] while generalized ones involve memory traces that might rely on cortical networks: at least this is what was observed in systems consolidation experiments, in which an engram became progressively independent of the hippocampus to be more permanently stored in neocortical areas $[[10,12,30-$ 33]. In a previous work, we showed that adult male rats exhibit both memory generalization and hippocampus-independence when tested 28 days after training, demonstrating that the loss of 
memory precision is a process that takes place concomitantly with the end of hippocampusdependence [4[4]is also consistent with other studies in the literature [6,13]. Here, we observed that in the adolescent male rats, memory generalization can be expressed earlier, 14 days after training, compared to male adults (Fig. 1C), an effect that, however, was not observed in adolescent females, suggesting a sex dimorphism (Fig. 1H-J).

Although we have studied animals in their late adolescence period [34-36], we cannot discard the possibility that the observed differences may be related to, say, the earlier sexual maturation of the females, which could have result in an accelerated maturation of relevant brain circuitry. In males, testosterone levels increase after day P40, reaching adult levels around P75 [25]. If we consider adult levels of gonadal-hormones as an optimum, these levels of testosterone may still not have been reached when subjects were trained in the contextual fear conditioning, and this could explain why a less detailed memory was formed. Moreover, the mean discrimination index indicates that adolescent male rats, when compared to females, display a lower discrimination level already after the most recent retrieval test, 2 days after learning (Fig. 1M).

On the other hand, acute and chronic administration of estrogen was shown to induce fear memory generalization in adult female rats $[17,18]$. Memory precision in adolescent female rats was found here to last longer (Fig. 1F-J;L), which could be explained by lower levels of estradiol in adolescents compared to adult animals. At the age these animals were trained (P42P49), they may yet not have reached typical adult levels of circulating estradiol [25], which was proven to accelerate memory generalization [15]. Along this line of thinking, ovariectomy should have resulted in a later onset of memory generalization, particularly due to reduced estrogen levels [35], however, in our experiments, ovariectomized females appear to have anticipated both systems consolidation and memory generalization, emulating their male counterparts (Fig. 3A-B). Previous studies demonstrated that gonadectomy prior to puberty can alter the organization of neural circuits [36] and modify behavioral expression [37,38]. In this way, we speculate that when ovariectomy is performed in an animal with an immature nervous system it could impair memory quality and somehow anticipate memory generalization.

\section{Conclusion}

Memory generalization and systems consolidation have distinct temporal courses in animals of different sex during adolescence. Memory generalization occurs earlier in adolescent 
males (14 days) than in females (28 days), the opposite of the observed in adult males, at least. Adolescent females appear to keep memory precision longer than males, and, in parallel, complete the systems consolidation process later. Ovariectomy, however, anticipated both memory generalization and retrieval hippocampus-independence in females, mimicking a male-like dynamics. 


\section{Role of authors}

wrote the manuscript: APC

edited the manuscript: FNL

reviewed the manuscript: JAQ, LOA

collected data: FNL, APC, MAC, KTKG, BP

\section{$\underline{\text { Histology }}$}

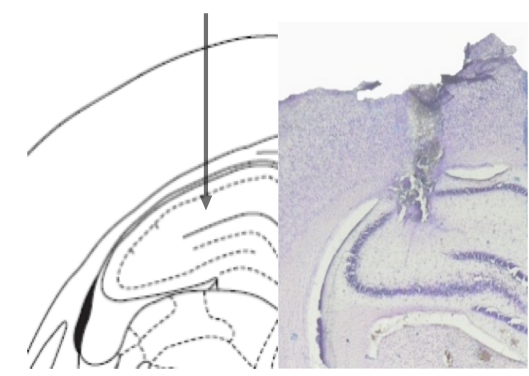

\section{Positions of the cannula tips}

\section{Fig. 2A}

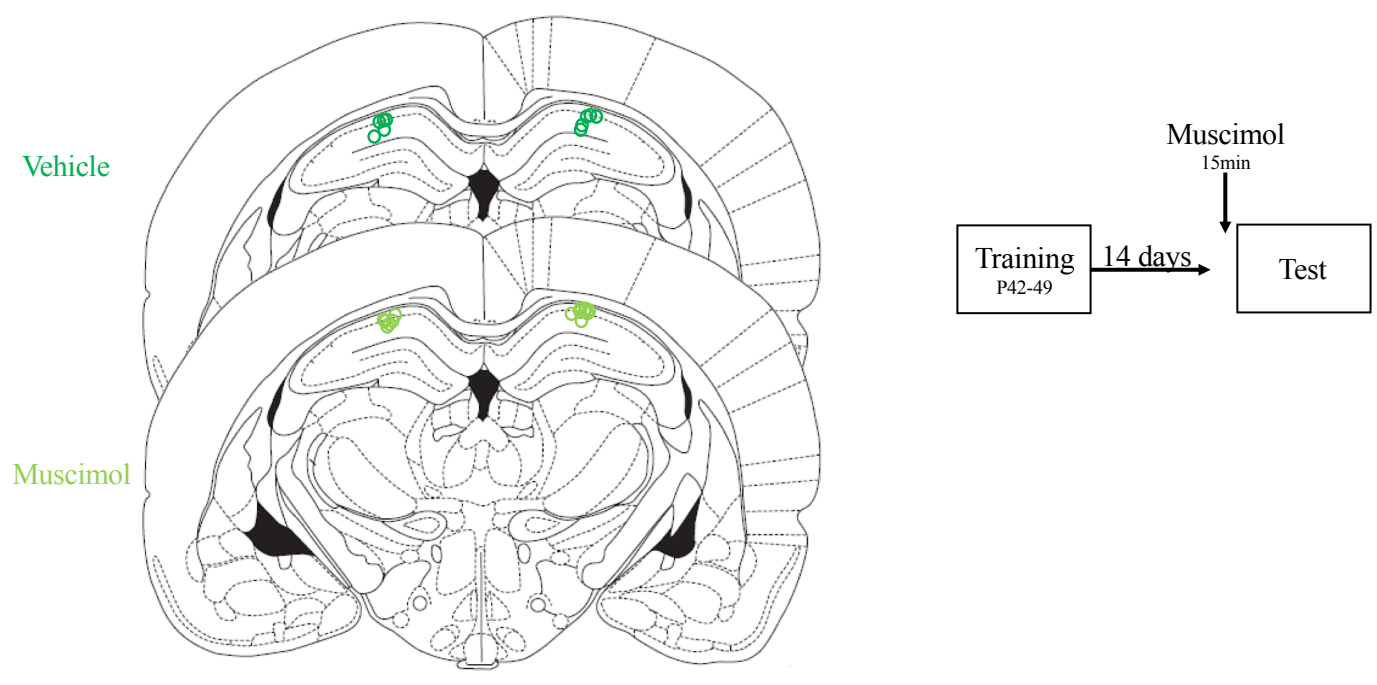




\section{Positions of the cannula tips}

\section{Fig. 2B}
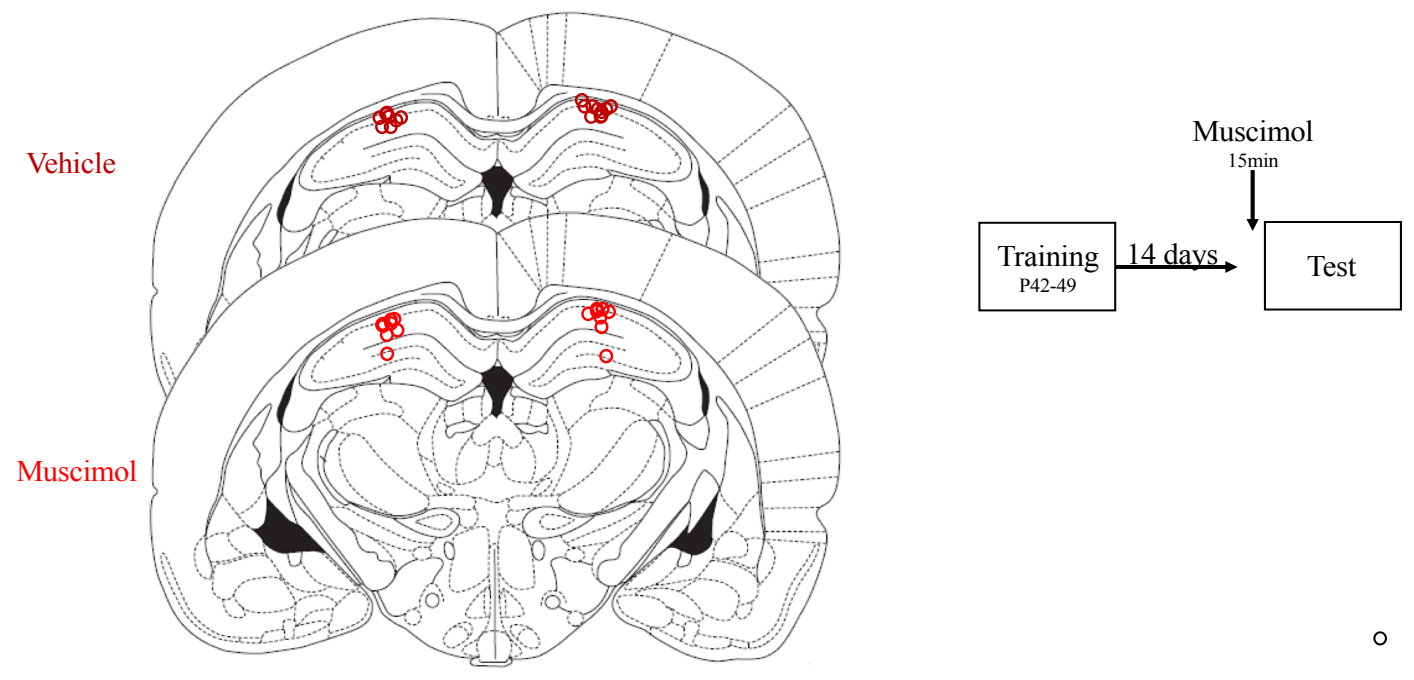

\section{Positions of the cannula tips}

\section{Fig. 3B}

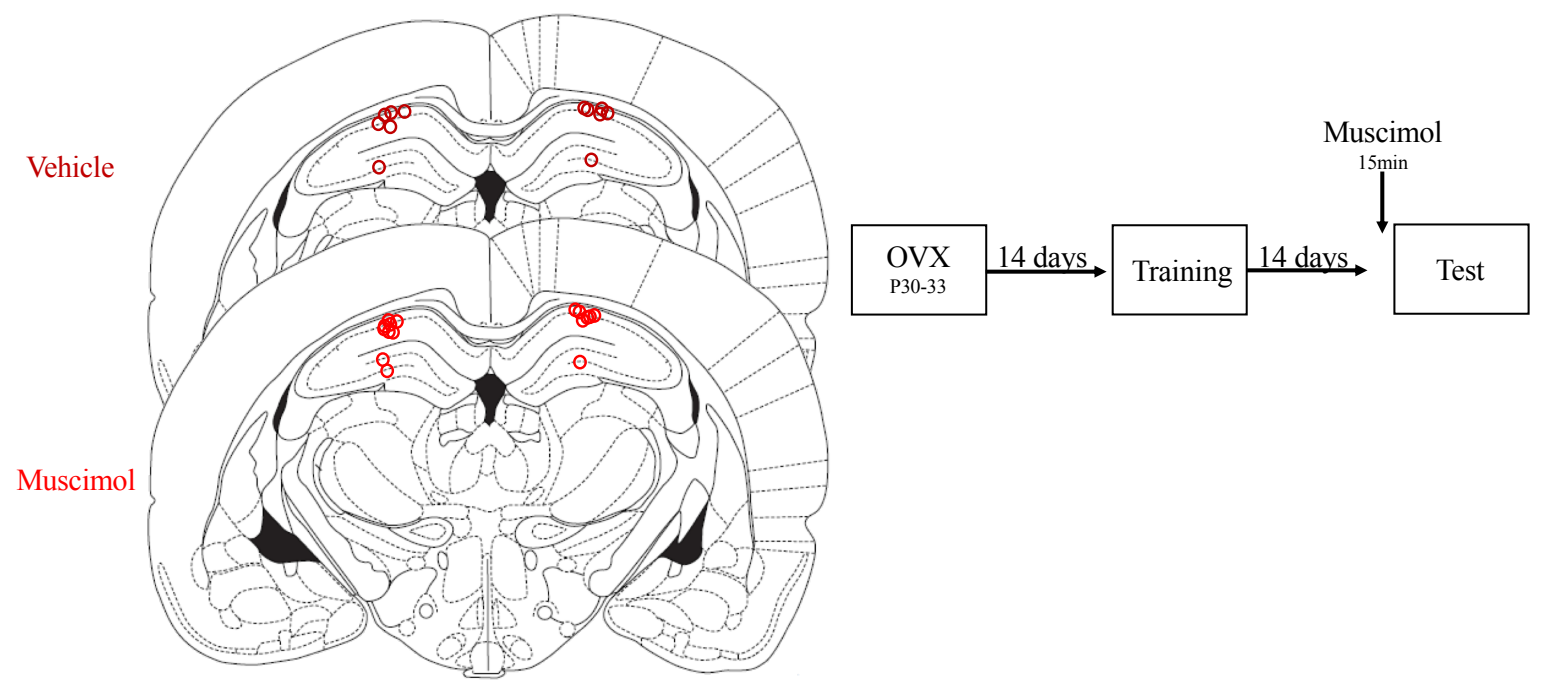

\section{Acknowledgements}

We thank Mrs. Zelma Regina V. de Almeida for her always kind and competent technical assistance. This work was supported by fellowships and grants from CAPES, CNPq and FAPERGS.

\section{Conflict of interests}

The authors declare that there is no conflict of interests. 


\section{References}

[1] J.W. Rudy, P. Morledge, Ontogeny of contextual fear conditioning in rats: implications for consolidation, infantile amnesia, and hippocampal system function., Behav. Neurosci. 108 (1994) 227-34. https://doi.org/10.1037//0735-7044.108.2.227.

[2] A.M. Jasnow, J.F.L. Iii, T.L. Gilman, D.C. Riccio, Review Perspectives on Fear Generalization and Its Implications for Emotional Disorders, 00 (2016). https://doi.org/10.1002/jnr.23837.

[3] L.P. Spear, The adolescent brain and age-related behavioral manifestations, Neurosci. Biobehav. Rev. (2000). https://doi.org/10.1016/S0149-7634(00)00014-2.

[4] L. de Oliveira Alvares, E.Ö. Einarsson, F. Santana, A.P. Crestani, J. Haubrich, L.F. Cassini, K. Nader, J.A. Quillfeldt, Periodically reactivated context memory retains its precision and dependence on the hippocampus, Hippocampus. 22 (2012) 1092-1095. https://doi.org/10.1002/hipo.20983.

[5] B.J. Wiltgen, A.J. Silva, Memory for context becomes less specific with time, Learn. Mem. (2007). https://doi.org/10.1101/1m.430907.

[6] B.J. Wiltgen, M. Zhou, Y. Cai, J. Balaji, M.G. Karlsson, S.N. Parivash, W. Li, A.J. Silva, The hippocampus plays a selective role in the retrieval of detailed contextual memories., Curr. Biol. 20 (2010) 1336-44. https://doi.org/10.1016/j.cub.2010.06.068.

[7] T. Maviel, T.P. Durkin, F. Menzaghi, B. Bontempi, Sites of neocortical reorganization critical for remote spatial memory, Science (80-. ). 305 (2004) 96-99. https://doi.org/10.1126/science.1098180.

[8] B. Bontempi, C. Laurent-Demir, C. Destrade, R. Jaffard, Time-dependent reorganization of brain circuitry underlying long-term memory storage., Nature. 400 (1999) 671-675. https://doi.org/10.1038/23270.

[9] P.W. Frankland, B. Bontempi, L.E. Talton, L. Kaczmarek, A.J. Silva, The Involvement of the Anterior Cingulate Cortex in Remote Contextual Fear Memory, Science (80-. ). (2004). https://doi.org/10.1126/science.1094804.

[10] J.A. Quillfeldt, M.S. Zanatta, P.K. Schmitz, J. Quevedo, E. Schaeffer, J.B. De Lima, J.H. Medina, I. Izquierdo, Different brain areas are involved in memory expression at different times from training, Neurobiol. Learn. Mem. (1996). https://doi.org/10.1006/nlme.1996.0050.

[11] R.E. Clark, N.J. Broadbent, L.R. Squire, Impaired remote spatial memory after hippocampal lesions despite extensive training beginning early in life, Hippocampus. 15 (2005) 340-346. https://doi.org/10.1002/hipo.20076.

[12] S.J. Martin, L. De Hoz, R.G.M. Morris, Retrograde amnesia: Neither partial nor complete hippocampal lesions in rats result in preferential sparing of remote spatial memory, even after reminding, Neuropsychologia. 43 (2005) 609-624. https://doi.org/10.1016/j.neuropsychologia.2004.07.007.

[13] A.P. Crestani, R.O. Sierra, A. Machado, J. Haubrich, K.M. Scienza, L. de Oliveira Alvares, J.A. Quillfeldt, Hippocampal plasticity mechanisms mediating experiencedependent learning change over time, Neurobiol. Learn. Mem. 150 (2018) 56-63. https://doi.org/10.1016/j.nlm.2018.02.020.

[14] P.K. Cullen, T.L. Gilman, P. Winiecki, D.C. Riccio, A.M. Jasnow, Activity of the anterior cingulate cortex and ventral hippocampus underlie increases in contextual fear generalization, Neurobiol. Learn. Mem. (2015). https://doi.org/10.1016/j.nlm.2015.07.001. 
[15] J. Lynch, P.K. Cullen, A.M. Jasnow, D.C. Riccio, Sex differences in the generalization of fear as a function of retention intervals, Learn. Mem. 20 (2013) 628-632. https://doi.org/10.1101/lm.032011.113.

[16] J.F. Lynch, P. Winiecki, T. Vanderhoof, D.C. Riccio, A.M. Jasnow, Hippocampal cytosolic estrogen receptors regulate fear generalization in females, Neurobiol. Learn. Mem. (2016). https://doi.org/10.1016/j.nlm.2016.01.010.

[17] K.P. Tolson, P.E. Chappell, The changes they are a-timed: Metabolism, endogenous clocks, and the timing of puberty, Front. Endocrinol. (Lausanne). (2012). https://doi.org/10.3389/fendo.2012.00045.

[18] C.R. Parker, V.B. Mahesh, Hormonal Events Surrounding the Natural Onset of Puberty in Female Rats1, Biol. Reprod. (1976). https://doi.org/10.1095/biolreprod14.3.347.

[19] D.L.M. Cyrenne, G.R. Brown, Effects of suppressing gonadal hormones on response to novel objects in adolescent rats, Horm. Behav. (2011). https://doi.org/10.1016/j.yhbeh.2011.08.015.

[20] B.J. Casey, R.M. Jones, T.A. Hare, The adolescent brain, Ann. N. Y. Acad. Sci. (2008). https://doi.org/10.1196/annals.1440.010.

[21] C.M. McCormick, I.Z. Mathews, Adolescent development, hypothalamic-pituitaryadrenal function, and programming of adult learning and memory, Prog. NeuroPsychopharmacology Biol. Psychiatry. (2010). https://doi.org/10.1016/j.pnpbp.2009.09.019.

[22] G.R. Brown, K.D. Kulbarsh, K.A. Spencer, C. Duval, Peri-pubertal exposure to testicular hormones organizes response to novel environments and social behaviour in adult male rats, Horm. Behav. (2015). https://doi.org/10.1016/j.yhbeh.2015.07.003.

[23] C.S. Vetter-O'Hagen, L.P. Spear, Hormonal and physical markers of puberty and their relationship to adolescent-typical novelty-directed behavior, Dev. Psychobiol. (2012). https://doi.org/10.1002/dev.20610.

[24] S.L. Andersen, Trajectories of brain development: point of vulnerability or window of opportunity?, Neurosci. Biobehav. Rev. 27 (2003) 3-18. https://doi.org/10.1016/S01497634(03)00005-8.

[25] T.L. Doremus-Fitzwater, M. Barreto, L.P. Spear, Age-related differences in impulsivity among adolescent and adult Sprague-Dawley rats, Behav. Neurosci. (2012). https://doi.org/10.1037/a0029697.

[26] S.B. Daughters, S.M. Gorka, A. Matusiewicz, K. Anderson, Gender specific effect of psychological stress and cortisol reactivity on adolescent risk taking, J. Abnorm. Child Psychol. (2013). https://doi.org/10.1007/s10802-013-9713-4.

[27] G. Paxinos, C. Watson, The Rat Brain in Stereotaxic Coordinates Sixth Edition, Elsevier Acad. Press. (2007).

[28] G. Winocur, M. Moscovitch, Memory transformation and systems consolidation, J. Int. Neuropsychol. Soc. (2011). https://doi.org/10.1017/S1355617711000683.

[29] G. Winocur, M. Moscovitch, M. Sekeres, Memory consolidation or transformation: context manipulation and hippocampal representations of memory, Nat. Neurosci. 10 (2007) 555-557. https://doi.org/10.1038/nn1880.

[30] Y. Dudai, R.G.M. Morris, Memorable trends, Neuron. 80 (2013) 742-750. https://doi.org/10.1016/j.neuron.2013.09.039.

[31] L.R. Squire, P. Alvarez, Retrograde amnesia and memory consolidation: a neurobiological perspective, Curr. Opin. Neurobiol. 5 (1995) 169-177. https://doi.org/10.1016/0959- 
4388(95)80023-9.

[32] L.R. Squire, C.E.L. Stark, R.E. Clark, The medial temporal lobe., Annu. Rev. Neurosci. 27 (2004) 279-306. https://doi.org/10.1146/annurev.neuro.27.070203.144130.

[33] L.R. Squire, L. Genzel, J.T. Wixted, R.G. Morris, Memory consolidation, Cold Spring Harb. Perspect. Biol. (2015). https://doi.org/10.1101/cshperspect.a021766.

[34] P. Sengupta, The laboratory rat: Relating its age with human's, Int. J. Prev. Med. (2013).

[35] L. Eiland, R.D. Romeo, Stress and the developing adolescent brain, Neuroscience. (2013). https://doi.org/10.1016/j.neuroscience.2012.10.048.

[36] S.J. Jackson, N. Andrews, D. Ball, I. Bellantuono, J. Gray, L. Hachoumi, A. Holmes, J. Latcham, A. Petrie, P. Potter, A. Rice, A. Ritchie, M. Stewart, C. Strepka, M. Yeoman, K. Chapman, Does age matter? The impact of rodent age on study outcomes, Lab. Anim. (2017). https://doi.org/10.1177/0023677216653984.

[37] W.A. Koss, M.M. Lloyd, R.N. Sadowski, L.M. Wise, J.M. Juraska, Gonadectomy before puberty increases the number of neurons and glia in the medial prefrontal cortex of female, but not male, rats, Dev. Psychobiol. (2015). https://doi.org/10.1002/dev.21290.

[38] J. Kercmar, T. Snoj, S.A. Tobet, G. Majdic, Gonadectomy prior to puberty decreases normal parental behavior in adult mice, Horm. Behav. (2014). https://doi.org/10.1016/j.yhbeh.2014.09.007.

[39] K. Delevich, C. Hall, J.R. Boivin, D. Piekarski, Y. Zhang, L. Wilbrecht, Prepubertal gonadectomy reveals sex differences in approach-avoidance behavior in adult mice, BioRxiv. (2019). https://doi.org/10.1101/638916. 